\title{
MODELO DE ONDAS CINEMÁTICAS NA SOLUÇÃO DO PROBLEMA INVERSO DA IRRIGAÇÃO POR SULCO EM TEMPO REAL
}

\author{
Carlos Alberto Vieira de Azevedo', Wynn R. Walker ${ }^{2}$ e Gary P. Merkley ${ }^{2}$
}

\begin{abstract}
RESUMO
Um modelo matemático computacional de ondas cinemáticas foi desenvolvido para interagir num processo de olimização não-lincar na solução do problema inverso da irrigação por sulco, que consiste na detcrminação das características de inlilltração d'água no solo, a partir dos dados da medição da láse de avanço. O modelo simula a fase de avanço para diferentes combinações entre os parâmetros de Kostiakov-Lewis, buscando minimizar as discrepâncias entre os tempos de avanço simulado e medido, e faz parte de um programa computacional, denominado SIRTOM (Surface Irrigation Real-Time Optimization Model) desenvolvido para solucionar o problema inverso. Baseando-se na solução inversa encontrada, SIRTOM faz prognósticos do desempenho do sistema de irrigação em tempo real, isto é, durante o evento. A habilidade do modelo em solucionar o problema inverso foi testada para difcrentes condições de campo. Eletivou-se o ajuste de avanços simulados com outros medidos em eventos de irrigação sob condições de fluxo contínuo e variável. Como inovação desta pesquisa, a estratégia de manejo com fluxo variável tem a finalidade de alterar a trajetória de avanço, quando detectado que a trajetória atual será uma catástrofe para o desempenho do sistema de irrigação. As análises demonstraram que o modelo de simulação desenvolvido nesta pesquisa é viável na solução do problema inverso, uma vę que, para lodas as condiçōes de campo estudadas, ele intcragiu satislatoriamente no processo de ajustagem. Constatou-se que a solução do problema inverso da irrigação por sulco é possível, mesmo durante estágios iniciais da fase de avanço, fato este que viabiliza ao programa SIRTOM fazer diagnósticos do desempenho do sistema de irrigação e sugerir, ao irrigante, alternativas para aprimorá-lo em tempo real.
\end{abstract}

Palavras-chave: sulco, avanço, desempenho, manejo de irrigação

\section{KINEMATIC-WAVE MODEL IN THE REAL-TIME SOLUTION OF THE INVERSE FURROW IRRIGATION PROBLEM}

\begin{abstract}
A kinematic-wave model was developed to interact upon a non-linear optimization process for solving the inverse furrow irrigation problem, which consists to determine the soil water infiltration characteristics from the advance phase measurements. The model simulates the advance phase for different combinations between the Kostiakov-Lewis parameters, purposing to minimize the discrepancies between the simulated and measured advance times. This model is part of a computer program, named SIRTOM (Surface Irrigation Real-Time Optimization Model), developed for solving the inverse problem. Based on the fund inverse solution, SIRTOM predicts the irrigation system performance on a real-time basis, that is, during the irrigation event. The model's ability to solve the inverse problem was tested under different field conditions. The simulated advance fittings were performed for advance phases measured in irrigation events under continuous and variable flow conditions. As an innovation of this research, the variable flow management strategy has the purpose to change the advance trajectory, whenever it would be detected that the current trajectory will be a
\end{abstract}

\footnotetext{
' Professor, PhD, Departamento de Engenharia Agrícola, Universidade Federal da Paraíba, Av. Aprigio Veloso 882, Bodocongó, CEP 58109-970, Campina Grande, PB, Brasil, e-mail: cazevedo@ deag.ufpb.br, Fone: (083) 310 1318, Fax: (083) 3101184. ${ }_{2}^{2}$ Prolessor, PhD, Department of Biological and Irrigation Enginecring, Utah Statc University, Logan, Utah, 84322-4105, USA, cmail: merkley@cc.usu.edu, Phone: (801) 797 2785, Fax: (801) 7971248.
} 
disaster for the irrigation system performance. The analysis showed that the simulation model developed in this research is viable in the solution of the inverse problem, since for all field conditions tested, it interacted satisfactorily upon the fitting process. It was verified that the solution for the furrow irrigation inverse problem is possible even during early stages of the advance phase, which substantially strengthens the SIRTOM program to make diagnosis of the irrigation system performance and to suggest for the irrigators alternatives for improving it in a real-lime basis.

Key words: furrow, advance, performance, irrigation management

\section{INTRODUÇÃO}

Os sistemas de irrigação superficiais têm o potencial de aplicar a água aos cultivos com eficiência de $70 \%$ a $85 \%$ (Merriam \& Keller, 1978; Willardson, 1972). Na verdade, alguns sistemas automatizados têm favorecido eficiências em torno de 90\% (Fischback \& Somerhalder, 1971); no entanto, a maioria dos projetos de irrigação por superfície no mundo tem trabalhado com eficiência de aplicação em torno de $40 \%$ a $50 \%$, ou com valores ainda menores (Bos \& Nugteren, 1974; Clyma el al., 1975; Kruse \& Heermann, 1977). Além de inadequado dimensionamento, outra causa para uma irrigação por superfície ineficiente está no manejo inapropriado.

O processo de dimensionamento e manejo dos sistemas de irrigação superficiais torna-se complicado devido às dificuldades que existem em se medir (ou estimar) as variações espaciais e temporais dos parâmetros de campo, especialmente as variações nas características de infiltração d'água no solo. O dimensionamento e o manejo dos referidos sistemas podem ser significativamente melhorados alravés da simulação matemálica do comportamento hidráulico de cada evento de irrigação sem, no entanto, requerer-se experimentações de campo extensivas, que são dispendiosas e consomem tempo (Elliott et al., 1983).

Os modelos hidráulicos da irrigação superficial foram originalmente desenvolvidos para simular as fases da irrigação e prognosticar os resultados qualitativos e quantitativos de um evento de irrigação, para um conjunto de parâmetros de campo conhecidos. A simulação, procedida desta forma, chama-se solução direta da irrigação superficial. A maneira como esses modelos foram concebidos tornou a solução direta tremendamente dependente da qualidade desses parâmetros de campo fornecidos a eles. Existem várias dificuldades em se quantificar tais parâmetros. A infiltração d'água no solo, em particular, apresenta-se como sendo a informação que mais compromete a simulação. Uma alternativa às medições de campo extensivas da infiltração, objetivando-se simular a fase de avanço, é solucionar o problema inverso da irrigação superficial, cujo avanço é medido e usado na simulação da infiltração.

Este trabalho teve o propósito de desenvolver um modelo matemático computacional, para simular a fase de avanço, capaz de interagir num processo de otimização não-linear de solução do problema inverso na irrigação por sulco em declive, tendo em vista o desenvolvimento de uma ferramenta computacional para tomada de decisão na melhoria do seu desempenho.

\section{DESENVOLVIMENTO TEÓRICO DO MODELO}

O modelo de ondas cinemáticas implementado nesta pesquisa baseou-se num desenvolvimento teórico apresentado por Walker \& Humpherys (1983). A equação da continuidade é descrita da seguinte forma:

$$
\frac{\partial A}{\partial t}+\frac{\partial Q}{\partial x}+\frac{\partial Z}{\partial \tau}=0
$$

em que A é a área da seção transversal de fluxo em $\mathbf{m}^{2}, \mathbf{t}$ é o tempo de ocorrência em minutos, $\mathbf{Q}$ é a vazão em $\mathbf{m}^{3} / \mathbf{m i n}, \mathbf{x}$ é a distância acumulada a partir da seção de entrada, em metros, $\boldsymbol{Z}$ é o volume infiltrado acumulado por unidade de comprimento de sulco em $\mathbf{m}^{3} / \mathbf{m}$, e t é o tempo de oportunidade de infiltração em minutos.

A equação de Manning foi utilizada, nesta análise, para gerar relação única entre vazão e seção hidráulica. Elliolt el al. (1982) propuseram uma relação empírica para a seção hidráulica dada por

$$
\left(A R^{2 / 3}\right)^{2}=\rho_{1} A^{\rho_{2}}
$$

Usando essa relação, a equação de Manning loma-se:

$$
Q=\alpha A^{m}
$$

onde:

$$
\begin{gathered}
\alpha=\frac{\sqrt{\rho_{1} S_{o}}}{n} . \\
m=\frac{\rho_{2}}{2}
\end{gathered}
$$

sendo n o coeficiente de rugosidade de Manning, $\mathbf{S}_{\mathbf{o}}$ a declividade do sulco, e $\rho_{1}$ e $\rho_{2}$ são constantes empíricas ajustadas às medições de campo atuais da geometria do sulco.

A Eq. 1 é integrada através de uma técnica de integração euleriana de primeira ordem para cada etapa de cálculo $(\delta \mathbf{t})$ e incremento espacial $(\delta \mathbf{x})$. Aplicando-se a integração, tem-se:

$$
\int_{i}^{t-\delta t}\left(\int_{x}^{x-\delta x} \frac{\partial Q}{\partial x} d x\right) d t+\int_{x}^{x-\delta x}\left(\int_{t}^{t+\delta t} \frac{\partial \Lambda}{\partial t} d t\right) d x+\int_{x}^{x+\delta x}\left(\int_{t}^{t+\delta x t} \frac{\partial Z}{\partial \tau} d t\right) d x=0
$$

O processo de integração resulta em:

$$
[\bar{Q}(x+\delta x, t)-\bar{Q}(x, t)] \delta t+[\bar{A}(x, t+\delta t)-\bar{A}(x, t)] \delta x+[\bar{Z}(x, t+\delta t)-\bar{Z}(x, t) \mid \delta x=0
$$

em que a barra sobre a variável de vazão (Q) indica ponderação temporal ao longo do intervalo de tempo dt e sobre as variáveis de área $(\mathbf{A})$ e infiltração acumulada $(\mathbf{Z})$ representa ponderações 
espaciais ao longo da distância dx.

Walker \& Humpherys (1983) desenvolveram os conceitos da Eq. 1, considerando o incremento do volume deformável d'água no sistema de fluxo correspondente a determinado período. Esse corpo deformável, ilustrado na Fig. 1, é designado, no sistema de fluxo, de célula. Cada célula na malha de cálculo, gerada no plano t-x (Fig. 2) pela técnica do controle de volume deformável, é identificada de acordo com as condições de contorno espacial e temporal; por exemplo, os subscritos J e $\mathbf{M}$ (Fig. 1) indicam, respectivamente, o tempo inicial e final de determinada célula, e os subscritos $\mathbf{L}$ e $\mathbf{R}$ referem-se aos contornos das células à montante e à jusante, respectivamente; assim, as condições de fluxo no contorno à monlante da célula são representadas por $\mathbf{J}$ e $\mathbf{L}$ e, no contorno à jusante, por $\mathbf{M}$ e R.

A Eq. 7 pode, então, ser escrila em termos da anotação apresentada na Fig. 1, tal como segue:

$$
\begin{aligned}
& \left\{\left[\theta Q_{R}+(1-\theta) Q_{M}\right]-\left[\theta Q_{I}+(1-\theta) Q_{I}\right]\right\} \delta t+ \\
& \left.\left\{\phi \Lambda_{I .}+(1-\phi) \Lambda_{R}\right]-\left[\phi \Lambda_{J}+(1-\phi) \Lambda_{M}\right]\right\} \delta x+ \\
& \left\{\phi Z_{J}+(1-\phi) Z_{R}\right]-\left[\phi Z_{L}+(1-\phi) Z_{M}\right] \delta \delta x=0
\end{aligned}
$$

sendo $\theta$ e $\phi$ coeficientes de ponderação temporal e espacial, respectivamente. Em geral, tem-se tomado valores iguais a 0,65 e 0,51 para $\theta$ e $\phi$, respectivamente.

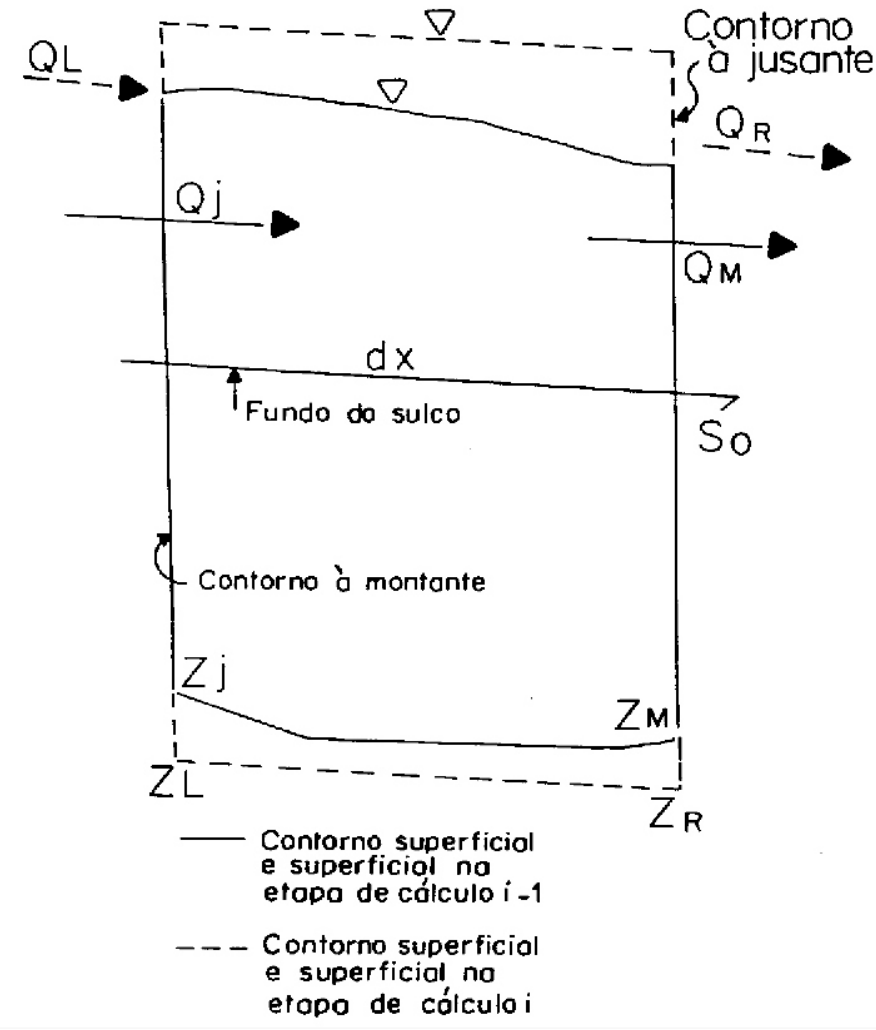

Figura 1. Célula computacional (Walker \& Humpherys, 1983).

\section{Solução numérica}

A solução é obtida resolvendo-se a Eq. 8 para cada célula na malha computacional (Fig. 2) começando-se horizontalmente da esquerda para a direita, em cada etapa do cálculo. As únicas incógnitas em cada célula são $\boldsymbol{Q}_{R}$ e $\boldsymbol{\Lambda}_{R^{-}}$. Substituindo-se a variável de vazão na Eq. 8 pela equação de Manning e se solucionando a referida equação para $\mathbf{A}_{\mathrm{k}}$, oblém-se a seguinte equação não-linear:

$$
\begin{gathered}
A_{R}^{m}+C_{1} A_{R}+C_{2}=0 \\
C_{1}=\left(\frac{1-\phi}{\alpha \theta}\right) \frac{\delta x}{\delta t}
\end{gathered}
$$

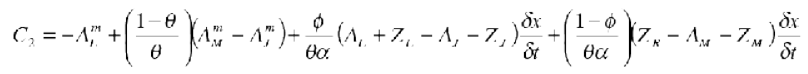

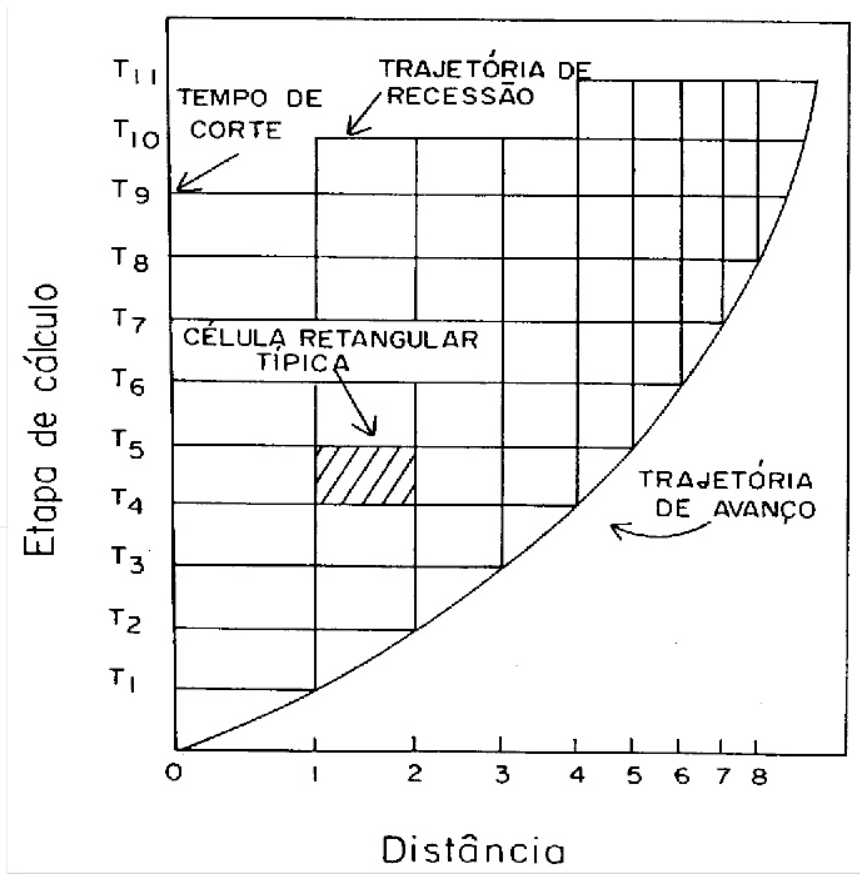

Figura 2. Malha computacional (Walker \& Humpherys, 1983).

\section{Condições iniciais}

As Equações 9 a 11 geram soluções para todas as células, excelo para aquelas na frente de avanço, onde $\mathbf{A}_{\mathrm{R}}, \mathbf{A}_{\mathrm{J}}, \mathbf{A}_{\mathrm{v}}, \mathbf{Z}_{\mathrm{R}}$, $\boldsymbol{Z}_{\mathbf{J}}$ e $\boldsymbol{T}_{\mathbf{M}}$ são iguais a zero. Para essas células, a formulação matemática das Equações 9 a 11 reduz-se a:

$$
-A_{L}^{m}+\frac{\phi}{\theta \alpha}\left(A_{L}+Z_{L}\right) \frac{\delta x_{1}}{\delta t}=0
$$

Uma vez que a única incógnita nas células da frente de avanço é a distância incremental (dx), a Eq. 12 torna-se:

$$
\delta x_{1}=\frac{\theta \alpha A_{L}^{m} \delta t}{\phi\left(A_{L}+Z_{L}\right)}
$$

\section{Condições de contorno}

O contorno à montante é definido pelas condições de fluxo na cabeceira da área irrigada, na qual $\mathbf{A}=\mathbf{0}$ e $\mathbf{Q}=\mathbf{0}$ para $\mathbf{t}=\mathbf{0}$ e $\mathbf{t}>\mathbf{t}_{\text {ap }}$ (tempo de aplicação) e $\boldsymbol{\Lambda}=\boldsymbol{\Lambda}_{\mathbf{0}}$ e $\mathbf{Q}_{\mathbf{0}}=\mathrm{a} \boldsymbol{\Lambda}^{\mathrm{m}}$ para $\mathbf{0}$ $<\mathbf{t} £ \mathbf{t}_{\mathrm{zp}}$. As condições de fluxo no contorno à jusante são descritas pelas Equações 3 e 13, respectivamente, para as fases de avanço e armazenamento, com ocorrência de escoamento superficial. 


\section{RESULTADOS E DISCUSSÃO}

\section{Validação do modelo}

O modelo foi verificado para quatro conjuntos de dados referentes às avaliações de campo de eventos de irrigação por sulco, publicados por Walker \& Humpherys (1983) e para seis numa trajelória de avanço medido em campo, é possível haver erros e variações nos parâmetros de campo, como também erros e tolerâncias inerentes ao modelo, que afetem as características de infiltração do solo, estimadas a partir do tipo de análise utilizada. A investigação da capacidade de simulação do modelo de ondas cinemáticas já foi feita por Reddell (1981), Sherman \& Singh (1982), Walker \& Humpherys (1983); verificou-se,
Tabela 1. Dados de campo utilizados na validação do modelo de simulação.

\begin{tabular}{|c|c|c|c|c|c|c|c|c|c|c|}
\hline \multirow{2}{*}{$\begin{array}{c}\text { Dados de } \\
\text { Campo }\end{array}$} & \multirow[b]{2}{*}{$F_{W F^{1}}$} & \multirow[b]{2}{*}{ FNWF' } & \multirow[b]{2}{*}{ KWFI } & \multirow[b]{2}{*}{$\mathrm{KNWF}^{1}$} & \multicolumn{3}{|c|}{ Fazenda Greenville } & \multicolumn{3}{|c|}{ Fazenda Amalga } \\
\hline & & & & & Guf-cq ${ }^{2}$ & Guf-iq ${ }^{2}$ & Guf-dq ${ }^{2}$ & Amal-cq ${ }^{2}$ & Amal-iq ${ }^{2}$ & Amal- $d q^{2}$ \\
\hline \multirow[t]{3}{*}{ Tipo de Solo } & Barto & Barro & Barro & Barro & Barro & Barro & Barro & Barro & Barro & Вагго \\
\hline & Arenoso & Arenoso & Sítico & Siltico & Síltico & Siltico & Siltico & Sfltico & Sítico & Sítico \\
\hline & & & Argiloso & Argiloso & & & & Argiloso & Argiloso & Argiloso \\
\hline Vazāo (l/s) & 2,0 & 2,0 & 1,5 & 0,8 & 1,3 & 0,52 & 1,2 & 1,8 & 0,82 & 1,83 \\
\hline Vazão & & & & & & 2,0 & 0,86 & & 2,0 & 1,14 \\
\hline alterada $(\mathrm{V} / \mathrm{s})^{3}$ & & & & & & & & & & \\
\hline Comprimento & 360 & 274 & 360 & 112 & 217 & 217 & 217 & 403 & 403 & 403 \\
\hline do sulco (m) & & & & & & & & & & \\
\hline Declivdade & 0,008 & 0,008 & 0,0104 & 0,0104 & 0,0173 & 0,0173 & 0,0173 & 0,0066 & 0,0066 & 0,0066 \\
\hline$(\mathrm{m} / \mathrm{m})$ & & & & & & & & & & \\
\hline Coeficiente & 0,013 & 0,013 & 0,013 & 0,013 & 0,013 & 0,013 & 0,013 & 0,013 & 0,013 & 0.013 \\
\hline \multicolumn{11}{|l|}{ dc Manning, } \\
\hline \multicolumn{11}{|l|}{ n } \\
\hline \multirow{2}{*}{\multicolumn{11}{|c|}{$\begin{array}{c}\text { Parâmetros } \\
\text { da Seçaảo }\end{array}$}} \\
\hline & & & & & & & & & & \\
\hline$\rho_{1}$ & 0,73 & 0,73 & 0.73 & 0,73 & 0,73 & 0.73 & 0,73 & 0,73 & 0,73 & 0,73 \\
\hline$\rho_{2}$ & 2,98 & 2,98 & 2,98 & 2,98 & 2,98 & 2,98 & 2,98 & 2,98 & 2,98 & 2,98 \\
\hline \multicolumn{11}{|l|}{ Distância de } \\
\hline Avanço ${ }^{4}(m)$ & & & & & & 93 & 93 & & 124 & 217 \\
\hline \multicolumn{11}{|l|}{ Infiltraçăo } \\
\hline \multicolumn{11}{|l|}{ medida } \\
\hline $\mathrm{k}\left(\mathrm{m}^{3} / \mathrm{min}^{2} / \mathrm{m}\right)$ & 0,0028 & 0,0022 & 0,0088 & & & & & & & \\
\hline 0 & 0,534 & 0,673 & 0,212 & & & & & & & \\
\hline fo $\left(\mathrm{m}^{3} / \mathrm{min} / \mathrm{m}\right)$ & 0,00022 & 0,00022 & 0,00017 & 0,00017 & 0,00022 & 0,00022 & 0,00022 & 0,00019 & 0,00019 & 0,00019 \\
\hline $\operatorname{Zreq}^{5}(\mathrm{~m})$ & 0,09 & 0,09 & 0,09 & 0,09 & 0,03 & 0,03 & 0,03 & 0,09 & 0,09 & 0,09 \\
\hline
\end{tabular}

'Dados publicados por Walker \& Humpherys (1983). As letras cq, iq e dq referem-se, respectivamente, à vazão constante, e a aumento e diminuiçăo de vazăo;

Dados publicados por Azevedo (1992):

Vazão variảvel durante o avanço;

4 Distáncia entre a seção de entrada e o local do avanço onde a vazão foi alterada

'Làmina d'água requerida.

conjuntos de dados coletados durante o estudo. A coleta de dados foi realizada em sistemas de irrigação por sulco existentes na propriedade $A M A L G A-U S A$ (privada) e na Estação Experimental da Utah State University-USA. Os dados coletados consistem de dois conjuntos para fluxo contínuo (Amalgacq e Gufcq) e de quatro conjuntos de dados para fluxo variável (Amalgaiq, Amalgadq, Gufiq e Gufdq). Foram medidas a vazão de entrada e a de saída (utilizando-se calhas tipo WSC), a perfilometria dos sulcos, a declividade do terreno e a fase de avanço. A Tabela 1 apresenta os dados de entrada para o modelo de ondas cinemáticas. Os dados dos avanços medidos estão apresentados mais adiante. Os conjuntos de dados FWF e KWF correspondem a sulcos compactados por roda de trator. $\mathrm{O}$ restante dos conjuntos de dados refere-se a sulcos não compactados. O sulco KNWF é um caso de variabilidade excessiva nos parâmetros de campo, que foi usado para se testar os limites do modelo na solução do problema inverso da irrigação por sulco.

Devido ao processo de solução do problema inverso da irrigação por sulco envolver a ajustagem de avanço simulado, porém, como o modelo se comporta na solução do problema inverso, principalmente diante de um avanço medido que agregue teor relativamente alto de variabilidade nos parâmetros de campo.

Aplicando-se a análise inversa ao avanço medido, as variações dos parâmetros de campo são incorporadas em apenas uma estimativa da infiltração, minimizando, consideravelmente, os impactos associados à solução do problema, o que se constitui numa grande vantagem desse tipo de análise. Por outro lado, a análise inversa durante a fase de avanço, cujos segmentos são usados para estimar a infiltração que será utilizada para prognosticar o tempo no qual ela terá seu término, os erros e variações nos dados de campo pode resultar em grandes variações nos parâmetros de infilltração estimados, de uma etapa de cálculo a outra.

$\mathrm{Na}$ solução do problema inverso, o avanço simulado é ajustado ao medido, objetivando-se determinar os parâmetros de infilltração para fins de estimativa do desempenho de um sistema de irrigação, num evento que possui determinadas características hidráulicas. A qualidade desse ajuste pode ser verificada tanto sob o aspecto prático da aplicação da solução inversa, como sob o ponto de vista de uma análise estatística. O critério fundamental na validação da solução inversa, sob o aspecto de sua utilidade prática, é o grau máximo permitido de discrepâncias entre o avanço simulado e o medido, para obter-se prognósticos confiáveis da eficiência de aplicação d'água. Para quantificar o grau de discrepâncias, elaborou-se uma função-objetivo, denominada resíduo, que corresponde à soma absoluta das diferenças entre o tempo de avanço simulado e o medido. A referida função é submetida ao processo de minimização pelo modelo de otimização não-linear desenvolvido por Azevedo (1992).

A Fig. 3 mostra, para os sulcos KNWF, KWF, FNWF, FWF e AMALGACQ, como o grau de discrepância afela os prognósticos da eficiência de aplicação. Nos sulcos KWF, FWF e AMALGACQ a eficiência de aplicação aumentou quando as discrepâncias diminuíram, porque o processo de tentativa da ajuslagem transcorreu de avanços mais lentos para avanços mais rápidos; fenômeno contrário ocorreu para os sulcos KNWF e FNWF. Observa-se que, após certo estágio do processo de 


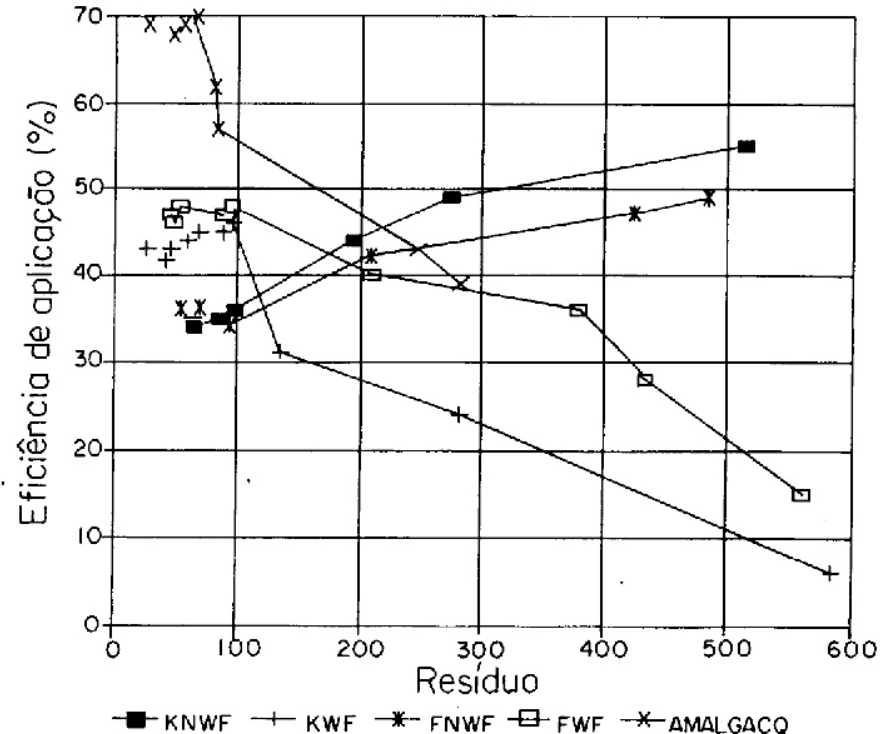

Figura 3. Discrepâncias entre os tempos de avanço simulado e medido versus eficiências de ampliação

minimização, decréscimos no resíduo alteraram, de forma insignificante, o valor da eficiência de aplicação; portanto, discrepâncias máximas permitidas são representadas pelo valor do resíduo, a partir do qual o fenômeno ocorre. O modelo detectou discrepâncias máximas permitidas num resíduo igual a 100 (Fig. 3); isto mostra que, para se obter prognósticos confiáveis do desempenho de um sistema de irrigação, o ajuste de avanços simulados não necessita apresentar alto grau de precisão.

Comparações entre trajelórias de avanços medidos e avanços simulados, a partir de valores dos parâmetros de Kostiakov-Lewis obtidos via processo de ajuste, são leitas nas Figuras 4 a 7 , respectivamente, para os sulcos $\mathbf{K W F}$, KNWF, FWF e FNWF. Essas Figuras ilustram o processo de ajustagem durante a fase de avanço, ou seja, são mostrados avanços simulados para diferentes percentagens do avanço medido utilizado no ajuste. Verifica-se que uma quantidade limitada de informações sobre o avanço medido, utilizada no ajuste, resulta em prognósticos da fase de avanço completa com maiores discrepâncias. A medida em que se aumenta a percentagem do avanço medido, as discrepâncias diminuem; entretanto, no sulco KWF um especílico trecho do avanço medido, quando adicionado na ajustagem, provocou aumento no resíduo; isto ocorreu, provavelmente, em virtude desse trecho agregar certo teor de variabilidade em parâmetros de campo ou porque acontece, nele, uma mudança brusca na declividade da trajetória de avanço, como consequência da redução do lluxo ao longo do sulco, devido à infiltração da água no solo.

De acordo com a análise prévia da Figura 3 , os ajustes apresentados nas Figuras 4 a 7 , que possuem resíduos inferiores a 100, resultam em soluções inversas aceitáveis. Questão fundamental é quando, durante a fase de avanço, uma solução aceitável pode lornar-se disponível para fïns de diagnóstico e controle do evento de irrigação. Verificou-se que a quantidade do avanço medido necessária à obtenção de uma solução aceitável está associada ao teor de variabilidade espacial em parâmetros de campo e ao grau de não-linearidade

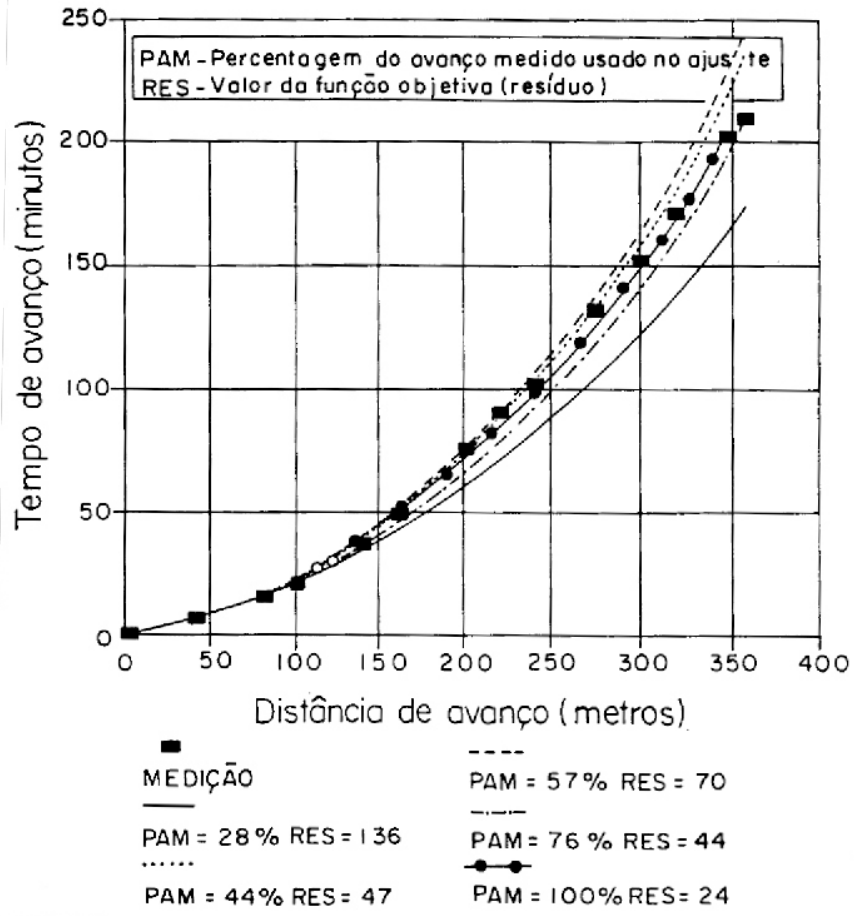

Figura 4. Ajuste, em tempo real, do avanço simulado para o sulco Kimberly Wheel (KMF).

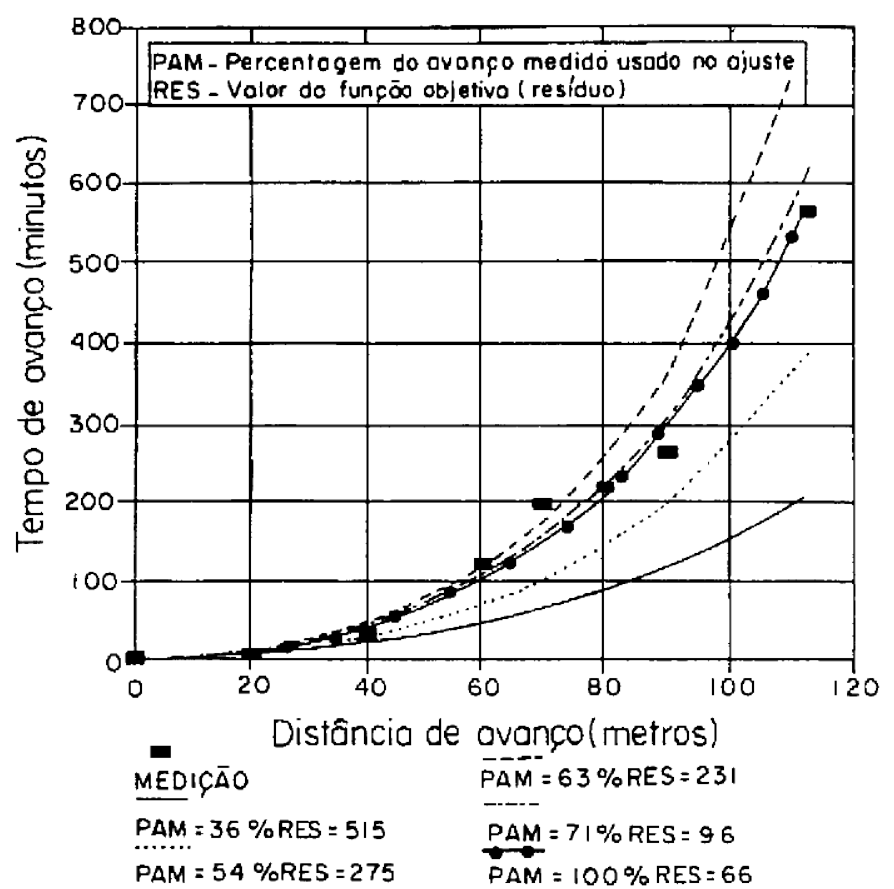

Figura 5. Ajuste, em tempo real, do avanço simulado para o sulco Kimberly Nonwheleel (KNWF).

na curva de avanço. O sulco KNWF incorpora variabilidade em parâmetros de campo muito acentuada; consequientemente, foi necessário que ocorressem $71 \%$ do avanço em campo para obtenção da solução aceitável. Como o sulco KWF apresenta não-linearidade suave, encontrouse uma solução aceitável em apenas $44 \%$ do avanço real; no entanto, o alto grau de não-linearidade nos sulcos FWF e FNWF só possibilitou a obtenção de soluções aceitáveis, 

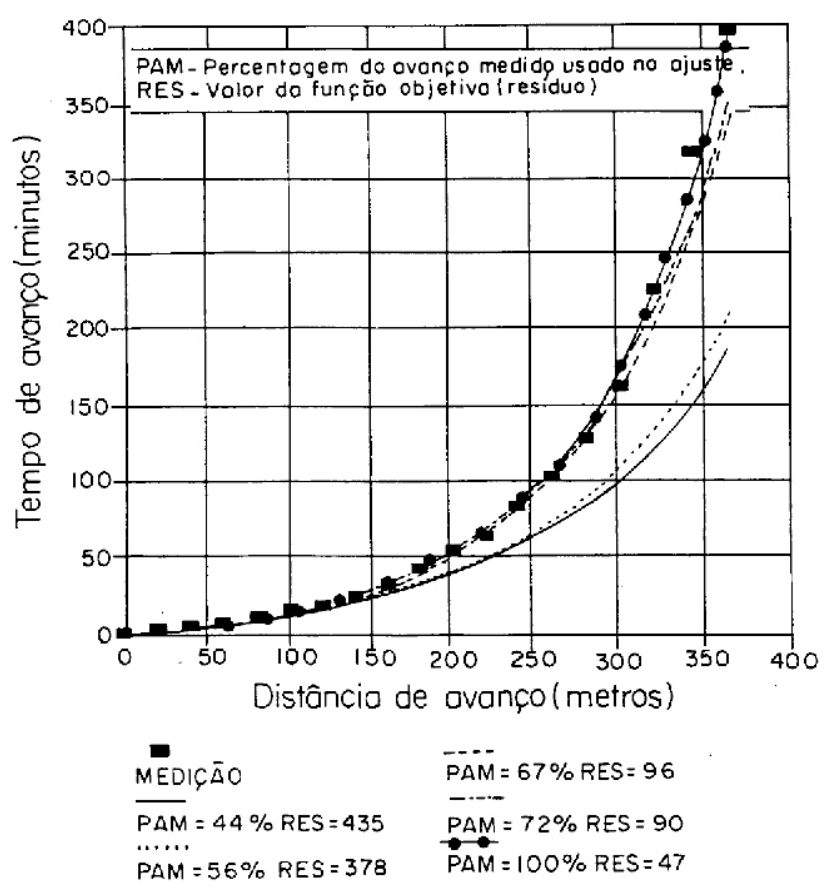

Figura 6. Ajuste, em tempo real, do avanço simulado para o sulco Flowewll Wheel (FWF).

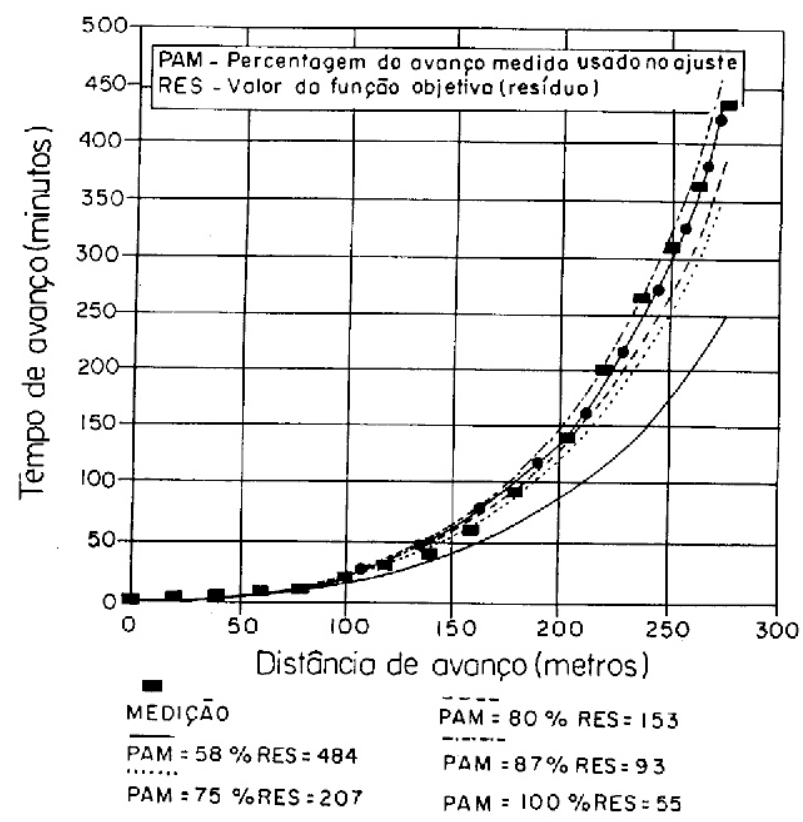

Figura 7. Ajuste, em tempo real, do avanço simulado para o sulco Flowewll Nonwheel (FNWF).

respectivamente, a partir de $67 \%$ e $87 \%$ do avanço no campo. Quanto mais cedo, durante a fase de avanço, se possa obter uma solução aceitável, maior será o trecho do avanço restante, que está para acontecer e que pode ser alterado para fins de melhoria do desempenho do sistema; entretanto, observou-se que prognósticos confiáveis da solução inversa durante estágios adiantados da fase de avanço podem, em alguns casos, ser ainda de grande valia para a melhoria do desempenho; por exemplo, o tempo de avanço no sulco FWF, a $67 \%$ de seu comprimento, correspondeu a apenas $21 \%$ do tempo de avanço no linal da área; isto demonstra que os danos no desempenho do sistema, nesse evento de irrigação, foram provocados mais pelo avanço da água que ocorreu no pequeno trecho de $67 \%$ a $100 \%$ do sulco, necessitando, então, ter sido alterado.

\section{CONCLUSÕES}

1. O ajuste de avanços simulados não necessita ser extremamente preciso para se ter prognósticos confiáveis do desempenho de um sistema de irrigação, o que torna possível as análises de identificação das características de infiltração do solo durante a fase de avanço.

2. O conhecimento a priori do grau máximo permitido de discrepâncias no ajuste possibilita que os resultados do processo de olimização se tornem disponíveis em tempo hábil, aspecto este imprescindível em sistemas de diagnóstico e controle, em tempo real, da irrigação.

3. Embora se tenha obtido solução aceitável para o sulco KNWF, avanços medidos, como o que agrega substancial variabilidade espacial em parâmetros de campo, poderão dificultar o processo de ajuste. Em tais circunstâncias, aconselhase que a ajuslagem no avanço simulado seja baseada num avanço medido que tenha sido devidamente ajustado a uma função potencial. Outro procedimento em situações como esta é fazerse com que o modelo de simulação trate as variações em declividade do terreno de forma mais judiciosa, qual seja, através de uma relação matemática.

4. Os sulcos estudados demonstraram que não se pode estabelecer previamente até onde o avanço deve ser medido para ter-se prognósticos de soluções aceitáveis; por conseguinte, estágios da fase de avanço devem ser medidos e incorporados ao processo de ajuste, até que a função-objetivo apresente valores inferiores a 100 .

5. O modelo de simulação desenvolvido nesta pesquisa é viável na solução do problema inverso da irrigação por sulco, uma vez que, para todas as condições de campo estudadas, ele interagiu satisfatoriamente no processo de ajustagem.

6. A solução do problema inverso da irrigação por sulco é possível, mesmo durante estágios iniciais da fase de avanço; isto torna mais ainda o programa SIRTOM, desenvolvido nesta pesquisa, capaz de diagnosticar e controlar as condiçōes hidráulicas de um evento de irrigação, tendo em vista aprimorar, em tempo real, o desempenho do sistema ao longo da estação de cultivo.

\section{REFERÊNCIAS BIBLIOGRÁFICAS}

AZEVEDO, C.A.V. de. Real-time solution of the inverse furrow advance problem. Logan: USU, 1992. 275p. Disserlação de Doutorado.

BOS, M.G., NUGTEREN, J. On irrigation efficiencies.

Publication n. 19. Wageningen: International Institute for Land Reclamation and Improvement, 1974. 64p.

CLYMA, W., ALI, A., ASHRAF, M.M. Irrigation practices and application efficiencies in Pakistan. Water Management Technical Report n. 39. Fort Collins : Colorado State University, 1975. 128p. 
ELLIOTT, R.L., WALKER W.R., SKOGERBOE G.V. Zeroinertia modeling of furrow irrigation advance. Journal of the Irrigation and Drainage Iivision, ASCE, New York, v. 108, n. IR3, p. 179-195, 1982.

ELLIOTT, R.L., WALKER, W.R., SKOGERBOE, G.V. Furrow irrigation advance rates: a dimensionless approach. Transactions of the ASAE, St. Joseph, v. 26, paper n. 822103 , p. $1722-1726,1983$

FISCHBACK, P.E., SOMERHALDER, B.R. Efficiencies of an automated surface irrigation system with and without a runoff reuse system. Transactions of the $\mathbf{A S} \mathbf{\Lambda} \mathbf{E}$, St. Joseph, v. 14, n. 4, p. 717-719, 1971.

KRUSE, E.G., HEERMANN, D.F. Implications of irrigations system efficiencies. Journal of Soil and Water Conservation, v. 32, n. 6, p. 465-270, 1977.
MERRIAM, J.L., KELLER, J. Farm irrigation system evaluation: a guide for management. Logan: Utah State University, 1978. 271p.

REDDELL, D.L. Modified rate of advance method for an automatic furrow irrigation system. Transactions of the ASA E, St. Joseph, v. 9, paper n. 81-2552, p. 552-556, 1981. SHERMAN, B., SINGH, V.P. A kinematic model for surface irrigation: an extension. Water Resources Research, v. 18, n. 3, p. 659-667, 1982.

WALKER, W.R., HUMPHERYS, A.S. Kinematic-wave furrow irrigation model. Journal of the Irrigation and Drainage Division, ASCE, New York, v. 109, n. 4, p. 377-392, 1983.

WILLARDSON, L.S. Allainable irrigation efficiencies. Journal of the Irrigation and Drainage Division, ASCE, New York, v. 98, n. IR2, p. $239-246,1972$. 